\title{
A Acupuntura é efetiva no tratamento das desordens temporomandibulares? Revisão da literatura
}

Is Acupuncture effective in the treatment of temporomandibular disorders? A literature review ¿Es la Acupuntura eficaz en el tratamiento de los transtornos. temporomandibulares? Una revisión de la literatura Mateus Silva Beker dos REIS

Faculdade de Odontologia, UNIFAL-MG Univ. Federal de Alfenas, 37133-840 Alfenas-MG, Brasil https://orcid.org/0000-0002-2240-6859

Ítalo Augusto Silva FERREIRA

Faculdade de Odontologia, UNIFAL-MG Univ. Federal de Alfenas, 37133-840 Alfenas-MG, Brasil https://orcid.org/0000-0002-6707-2178 Jader Bueno DE OLIVEIRA

Faculdade de Odontologia, UNIFAL-MG Univ. Federal de Alfenas, 37133-840 Alfenas-MG, Brasil https://orcid.org/0000-0002-1702-7416 Letícia da Costa SIQUEIRA

Faculdade de Odontologia, UNIFAL-MG Univ. Federal de Alfenas, 37133-840 Alfenas-MG, Brasil https://orcid.org/0000-0003-0048-1273 Loren dos Santos ANDRADE

Faculdade de Odontologia, UNIFAL-MG Univ. Federal de Alfenas, 37133-840 Alfenas-MG, Brasil https://orcid.org/0000-0002-7071-6296 Vinícius Ribeiro MONTEIRO

Faculdade de Odontologia, UNIFAL-MG Univ. Federal de Alfenas, 37133-840 Alfenas-MG, Brasil https://orcid.org/0000-0002-2798-6311

Marcela Filié HADDAD

Faculdade de Odontologia, UNIFAL-MG Univ. Federal de Alfenas, 37133-840 Alfenas-MG, Brasil https://orcid.org/0000-0003-3455-6624

\section{Resumo}

Introdução: A disfunção temporomandibular é um grupo de condições que apresentam como sintoma a dor na região da articulação temporomandibular. A etiologia dessa condição ainda é incerta, mas é sugerida como multifatorial. O foco principal no tratamento da disfunção temporomandibular é o controle da dor, e da disfunção do aparelho mastigatório de modo a atenuar cargas adversas que acabam perpetuando o problema. A acupuntura faz parte da Medicina Tradicional Chinesa, técnica que consiste basicamente na introdução de agulhas em pontos específicos da superfície corporal, e tem sido usada para promover o alívio da dor. Objetivo: avaliar a utilização da acupuntura no tratamento de desordens temporomandibulares. Materiais e métodos: Foi realizada uma revisão de literatura com busca nas bases dados PubMed, Lilacs, Medline e BBO no período de 2000 a 2020. Resultados: Foram encontrados 52 artigos, excluídos 37 artigos após a leitura de títulos e resumos, sendo incluídos 15 artigos. Conclusão: A partir da literatura pesquisada, pode-se concluir que a acupuntura é uma terapia de suporte complementar no tratamento das disfunções temporomandibulares e tem se mostrado tão eficiente no controle de dores faciais quanto as terapias convencionais.

Descritores: Acupuntura; Terapia por Acupuntura; Dor Facial; Síndrome da Disfunção da Articulação Temporomandibular.

\section{Abstract}

Temporomandibular disorder (TMD) is a group of conditions that present pain in the temporomandibular joint (TMJ) region as a symptom. The etiology of this condition is still uncertain, but it is suggested as multifactorial. The main focus in the treatment of TMD is the control of pain, and the dysfunction of the masticatory apparatus in order to mitigate adverse loads that end up perpetuating the problem. Acupuncture is part of Traditional Chinese Medicine, a technique that basically consists of introducing needles at specific points on the body surface, and has been used to promote pain relief. This study aimed to evaluate the use of acupuncture in the treatment of TMDs. A literature review was carried out with search in the databases PubMed, Lilacs, Medline and BBO in the period from 2000 to 2020 so that 53 articles were found. Thirty seven articles were excluded after reading titles and abstracts, including 16 articles. From the researched literature, it can be concluded that acupuncture is a complementary supportive therapy in the treatment of temporomandibular disorders and has been shown to be as efficient in controlling facial pain as conventional therapies.

Descriptors: Acupuncture; Acupuncture Therapy; Facial Pain; Temporomandibular Joint Dysfunction Syndrome.

\section{Resumen}

Introducción: El trastorno temporomandibular es un grupo de afecciones que presentan como síntoma dolor en la articulación temporomandibular. La etiología de esta condición aún es incierta, pero se sugiere como multifactorial. El principal foco en el tratamiento de los trastornos temporomandibulares es el control del dolor y la disfunción del aparato masticatorio para mitigar cargas adversas que terminan perpetuando el problema. La acupuntura forma parte de la Medicina Tradicional China, una técnica que básicamente consiste en introducir agujas en puntos específicos de la superficie corporal, y se ha utilizado para favorecer el alivio del dolor. Objetivo: evaluar el uso de la acupuntura en el tratamiento de trastornos temporomandibulares. Materiales y métodos: Se realizó una revisión de la literatura con una búsqueda en las bases de datos PubMed, Lilacs, Medline y BBO en el período de 2000 a 2020. Resultados: Se encontraron 52 artículos, 37 artículos fueron excluidos luego de la lectura de títulos y resúmenes, incluyendo 15 artículos. Conclusión: De la literatura investigada se puede concluir que la acupuntura es una terapia de apoyo complementario en el tratamiento de los trastornos temporomandibulares y ha demostrado ser tan eficaz en el control del dolor facial como las terapias convencionales.

Descriptores: Acupuntura; Terapia por Acupuntura; Dolor Facial; Síndrome de la Disfunción de Articulación Temporomandibular.

INTRODUÇÃO

A disfunção temporomandibular (DTM) diz respeito a um grupo de condições que apresentam a dor como característica principal. Atinge a articulação temporomandibular (ATM), os músculos mastigatórios e as demais estruturas associadas. Pode apresentar sinais clínicos como limitação da abertura bucal, cliques na ATM e crepitação. Ademais, alguns pacientes podem relatar sintomas como tontura e zumbido $3,4,11,15$. 
A etiologia dessa condição ainda não é bem estabelecida na literatura, mas é sugerida como multifatorial e relacionada a diversos fatores físicos, psicossociais e funcionais, como alterações na oclusão, anormalidades no disco intra-articular, hábitos parafuncionais, macrotraumas na região orofacial, estresse, alterações hormonais e alterações posturais $^{3,4,11}$.

O tratamento da DTM é focado no controle da dor e da disfunção do aparelho mastigatório e visa atenuar cargas adversas que acabam mantendo o problema. As abordagens terapêuticas iniciais se dão por meio de terapias reversíveis como o uso de placas oclusais miorrelaxantes, utilização de medicamentos, fisioterapia envolvendo ou não calor, controle do estresse, treinamento postural e reeducação do paciente. Nos casos mais complexos onde os tratamentos não invasivos são ineficazes, optase por terapias invasivas e irreversíveis como ajuste oclusal por desgaste seletivo, terapia ortodôntica, cirurgia ortognática ou técnicas de reabilitação oral protética. Existem ainda, métodos complementares como, injeção de toxina botulínica e a acupuntura e suas diversas formas de aplicação $0^{3,14}$.

A acupuntura faz parte da Medicina Tradicional Chinesa, ciência que tem registros de mais de três mil anos no Oriente. Esta técnica consiste, basicamente, na introdução de agulhas em pontos específicos da superfície corporal, e tem sido usada para promover o alívio da dor. Acredita-se que a sua aplicação ative um mecanismo de modulação da dor, através da liberação de endorfinas e neurotransmissores ${ }^{8,13,14,16}$. A literatura mostra diversos estudos que comprovam a eficácia do uso da acupuntura na odontologia. Essa técnica tem sido benéfica no tratamento da disfunção dos músculos mastigatórios e na articulação temporomandibular, nas dores odontogênicas, nas neuralgias trigeminais idiopáticas, nas sinusites maxilares e em casos de abertura limitada de boca ${ }^{10,13,16}$. O objetivo do presente trabalho foi avaliar por meio de uma revisão de literatura, a aplicação da acupuntura no tratamento das DTMs.

MATERIAL E MÉTODO

Esse trabalho foi elaborado a partir de revisão de literatura nas bases de dados PubMed, Lilacs, Medline e BBO no período de 2000 a 2020. As palavras-chave utilizadas foram "Acupunture", "Acupuncture Therapy", "Masticatory Muscles", "Temporomandibular Joint Dysfunction Syndrome", "Facial Pain", "Acupuncture Analgesia", "Temporomandibular Joint Disorders", "Acupuncture Points" e "Trigger
Points". Foram critérios de exclusão artigos publicados antes de 2000 ou que não se correlacionavam à aplicação da acupuntura no tratamento das desordens temporomandibulares.

No total foram encontrados 52 artigos. Após a leitura dos títulos e resumo foram excluídos 37 deles, pois notou-se que alguns se repetiram nas diferentes bases de dados e outros não correlacionavam 0 uso da acupuntura no tratamento das desordens temporomandibulares. Foram incluídos 15 trabalhos na forma de revisão de literatura, relato de caso, ensaio clínico e estudo retrospectivo que correlacionavam o uso da acupuntura e o tratamento das DTMs.

REVISÃO DA LITERATURA

Os quinze trabalhos selecionados para compor essa revisão foram publicados no período de 2000 a 2013. Foram encontrados onze estudos avaliando a acupuntura tradicional no tratamento da sintomatologia dolorosa da DTM; Três trabalhos analisando o efeito da acupuntura a laser; Um estudo avaliando o uso de fitoterapia; Um estudo investigando a eficácia do agulhamento à seco.

Tabela 1. Características dos artigos incluídos no estudo

\begin{tabular}{|c|c|c|c|}
\hline Autor/ano & Objetivo do trabalho & $\begin{array}{c}\text { Técnica } \\
\text { utilizada }\end{array}$ & Resultado obtido \\
\hline $\begin{array}{l}\text { Rosted } \\
(2000)\end{array}$ & $\begin{array}{c}\text { Apresentar pesquisas e estudos } \\
\text { relevantes que mostram o valor } \\
\text { da acupuntura para o } \\
\text { tratamento das DTMs e dor } \\
\text { facial. } \\
\end{array}$ & $\begin{array}{l}\text { Acupuntura } \\
\text { Tradicional. }\end{array}$ & $\begin{array}{c}\text { A acupuntura deve ser } \\
\text { considerada tratamento } \\
\text { complementar ao } \\
\text { convencional para as } \\
\text { DTMs. } \\
\end{array}$ \\
\hline $\begin{array}{c}\text { Rosted e } \\
\text { Jorgensen } \\
(\mathbf{2 0 0 2 )}\end{array}$ & $\begin{array}{c}\text { Relato de caso do uso da } \\
\text { acupuntura como tratamento } \\
\text { para DTM muscular em estágio } \\
\text { inicial. }\end{array}$ & $\begin{array}{l}\text { Acupuntura } \\
\text { Tradicional. }\end{array}$ & $\begin{array}{l}\text { A acupuntura deve ser } \\
\text { considerada como } \\
\text { tratamento em estágios } \\
\text { iniciais de restrição nos } \\
\text { movimentos da ATM. }\end{array}$ \\
\hline $\begin{array}{c}\text { Schmid- } \\
\text { Schwap et al. } \\
\quad(2006)\end{array}$ & $\begin{array}{c}\text { O estudo teve como objetivo } \\
\text { avaliar os } \\
\text { efeitos da acupuntura em } \\
\text { pacientes com disfunção } \\
\text { temporomandibular em } \\
\text { comparação à terapia com } \\
\text { placebo } \\
\text { (laser); o parâmetro alvo } \\
\text { primário foi a } \\
\text { sensação de dor. }\end{array}$ & $\begin{array}{l}\text { Acupuntura } \\
\text { Tradicional } \\
\text { (Grupo 1) e } \\
\text { Acupuntura } \\
\text { a Laser } \\
\text { Simulada } \\
\text { (Grupo 2). }\end{array}$ & $\begin{array}{l}\text { A acupuntura pode ser } \\
\text { recomendada como } \\
\text { tratamento para distúrbios } \\
\text { craniomandibulares } \\
\text { agudos. Mas estudos com } \\
\text { maior número de } \\
\text { pacientes são necessários } \\
\text { para investigar o } \\
\text { tratamento a longo prazo }\end{array}$ \\
\hline $\begin{array}{l}\text { Rosted et al. } \\
\text { (2006) }\end{array}$ & $\begin{array}{l}\text { Determinar se os resultados do } \\
\text { uso da acupuntura para } \\
\text { tratamento da disfunção } \\
\text { temporomandibular são } \\
\text { comparáveis aos resultados } \\
\text { obtidos em estudos anteriores } \\
\text { feitos em clínicas } \\
\text { universitárias. }\end{array}$ & $\begin{array}{c}\text { Acupuntura } \\
\text { Tradicional } \\
\text { e } \\
\text { Estimulação } \\
\text { Manual. }\end{array}$ & $\begin{array}{c}\text { Os resultados do uso da } \\
\text { acupuntura } \\
\text { no tratamento da DTM na } \\
\text { prática odontológica geral } \\
\text { são comparáveis aos } \\
\text { obtidos em estudos } \\
\text { clínicos em ambientes } \\
\text { universitários. Sendo } \\
\text { assim, é considerado um } \\
\text { método simples, } \\
\text { relativamente seguro e } \\
\text { potencialmente eficaz e } \\
\text { útil na gestão } \\
\text { de DTM. }\end{array}$ \\
\hline $\begin{array}{l}\text { Shin et al. } \\
(2007)\end{array}$ & $\begin{array}{l}\text { Estudo retrospectivo de uma } \\
\text { série de casos investigou os } \\
\text { efeitos da combinação de } \\
\text { terapia manual e acupuntura } \\
\text { na dor associada } \\
\text { à DTM. }\end{array}$ & $\begin{array}{l}\text { Acupuntura } \\
\text { Tradicional } \\
\text { e Terapia } \\
\text { Manual. }\end{array}$ & $\begin{array}{c}\text { Forneceu uma base para a } \\
\text { exploração de } \\
\text { combinações } \\
\text { terapia manual e } \\
\text { acupuntura como terapia } \\
\text { não farmacológica para } \\
\text { reduzir a dor e aumentar a } \\
\text { abertura máxima bucal } \\
\text { de pacientes com DTM. }\end{array}$ \\
\hline $\begin{array}{l}\text { Shen et al. } \\
\text { (2007) }\end{array}$ & $\begin{array}{l}\text { Avaliar a curto prazo os efeitos } \\
\text { da acupuntura no tratamento } \\
\text { da dor miofascial em } \\
\text { decorrência da DTM. }\end{array}$ & $\begin{array}{l}\text { Acupuntura } \\
\text { Tradicional. }\end{array}$ & $\begin{array}{l}\text { O estudo concluiu que a } \\
\text { acupuntura aumentou } \\
\text { significativamente a } \\
\text { tolerância à dor do } \\
\text { músculo masseter }(\mathrm{P}= \\
\text { o,o27). Também } \\
\text { encontrou evidências de } \\
\text { efeito placebo quando a } \\
\text { percepção de receber } \\
\text { acupuntura reduziu } \\
\text { significativamente a dor } \\
\text { facial }(\mathrm{P}=\mathrm{O}, \mathrm{OOO}), \\
\text { dor no pescoço }(\mathrm{P}=\mathrm{O}, \mathrm{o11}) \\
\text { e dor de cabeça }(\mathrm{P}= \\
\mathrm{O}, \mathrm{O15}) .\end{array}$ \\
\hline
\end{tabular}


Tabela 1 (Continuação). Características dos artigos incluídos no estudo

\begin{tabular}{|c|c|c|c|}
\hline Autor/ano & Objetivo do trabalho & $\begin{array}{c}\text { Técnica } \\
\text { utilizada }\end{array}$ & Resultado obtido \\
\hline $\begin{array}{l}\text { Smith et al. } \\
(2007)\end{array}$ & $\begin{array}{l}\text { Comparar o efeito da } \\
\text { acupuntura real e da } \\
\text { acupuntura simulada no } \\
\text { tratamento da dor miofascial } \\
\text { da articulação } \\
\text { temporomandibular, a fim de } \\
\text { estabelecer a verdadeira } \\
\text { eficácia da } \\
\text { Acupuntura. }\end{array}$ & $\begin{array}{l}\text { Acupuntura } \\
\text { Tradicional. }\end{array}$ & $\begin{array}{c}\text { A acupuntura teve uma } \\
\text { influência positiva nos } \\
\text { sinais e sintomas da dor } \\
\text { miofascial em decorrência } \\
\text { da DTM. }\end{array}$ \\
\hline $\begin{array}{l}\text { Bergstrom et } \\
\text { al. (2008) }\end{array}$ & $\begin{array}{c}\text { Avaliar a presença de } \\
\text { sintomas de desordens } \\
\text { temporomandibulares } \\
\text { (DTMs) em pacientes } \\
\text { encaminhados para } \\
\text { clínica especializada devido a } \\
\text { problemas musculares e que } \\
\text { haviam recebido } \\
\text { principalmente acupuntura e } \\
\text { / ou } \\
\text { terapia com aparelho } \\
\text { interoclusal. }\end{array}$ & $\begin{array}{c}\text { Acupuntura } \\
\text { Tradicional e } \\
\text { Terapia } \\
\text { Interoclusal }\end{array}$ & $\begin{array}{c}\text { A maioria dos pacientes } \\
\text { relatou uma melhora } \\
\text { duradoura em seus } \\
\text { sintomas após a associação } \\
\text { das terapias. }\end{array}$ \\
\hline$\underset{(2009)}{\text { Simma et al. }}$ & $\begin{array}{c}\text { Estudar os efeitos imediatos } \\
\text { da acupuntura em pacientes } \\
\text { com distúrbios } \\
\text { craniomandibulares. }\end{array}$ & $\begin{array}{c}\text { Acupuntura } \\
\text { Tradicional e } \\
\text { Acupuntura } \\
\text { a Laser } \\
\text { Simulada }\end{array}$ & $\begin{array}{l}\text { Achados após a realização } \\
\text { da acupuntura } \\
\text { demonstraram maior } \\
\text { redução da dor quando } \\
\text { comparado com o placebo } \\
\text { (p=o,o31). Em } \\
\text { comparação com o laser, a } \\
\text { acupuntura pode trazer } \\
\text { alívio imediato da dor. }\end{array}$ \\
\hline $\begin{array}{l}\text { Katsoulis } \\
\text { et al. } \\
(\mathbf{2 0 1 0})\end{array}$ & $\begin{array}{l}\text { Avaliar a eficácia da } \\
\text { acupuntura a laser. }\end{array}$ & $\begin{array}{l}\text { Acupuntura } \\
\text { a Laser e } \\
\text { Acupuntura } \\
\text { a Laser } \\
\text { Simulada. }\end{array}$ & $\begin{array}{c}\text { Devido ao baixo número } \\
\text { de participantes nenhuma } \\
\text { conclusão clara pode ser } \\
\text { tirada. A acupuntura a } \\
\text { laser pode ser um } \\
\text { tratamento opcional para } \\
\text { pacientes com interesse } \\
\text { em um tratamento } \\
\text { complementar não } \\
\text { invasivo. } \\
\end{array}$ \\
\hline $\begin{array}{l}\text { Hotta et al. } \\
\text { (2010) }\end{array}$ & $\begin{array}{l}\text { Analisar o efeito do laser de } \\
\text { baixo nível aplicado aos } \\
\text { pontos de acupuntura dos } \\
\text { pacientes diagnosticado } \\
\text { com disfunção } \\
\text { temporomandibular. }\end{array}$ & $\begin{array}{l}\text { Acupuntura } \\
\text { a Laser. }\end{array}$ & $\begin{array}{c}\text { Os testes estatísticos } \\
\text { mostram melhorias } \\
\text { significativas (p<0,01) em } \\
\text { sintomas dolorosos e } \\
\text { eletromiográficos, mas em } \\
\text { medições de movimentos } \\
\text { mandibulares não } \\
\text { demonstrou melhorias } \\
\text { significativas (p } 1 / 40,05) \text {. }\end{array}$ \\
\hline $\begin{array}{c}\text { Hunter } \\
\text { (2011) }\end{array}$ & $\begin{array}{l}\text { Analisar o uso da } \\
\text { acupuntura para } \\
\text { tratamento em um músico } \\
\text { profissional com disfunção } \\
\text { temporomandibular. }\end{array}$ & $\begin{array}{l}\text { Acupuntura } \\
\text { Tradicional. }\end{array}$ & $\begin{array}{l}\text { Após o tratamento houve } \\
\text { remissão dos sintomas de } \\
\text { dor do paciente. }\end{array}$ \\
\hline $\begin{array}{l}\text { Ritenbaugh } \\
\text { et al. } \\
(\mathbf{2 0 1 2})\end{array}$ & $\begin{array}{c}\text { Este estudo procurou } \\
\text { identificar o papel } \\
\text { apropriado para a medicina } \\
\text { tradicional chinesa } \\
\text { (acupuntura e ervas) em } \\
\text { conjunto com uma } \\
\text { intervenção validada de } \\
\text { autocuidado psicossocial } \\
\text { para o tratamento de } \\
\text { doenças } \\
\text { temporomandibulares } \\
\text { crônicas. }\end{array}$ & $\begin{array}{l}\text { Acupuntura } \\
\text { Tradicional e } \\
\text { Ervas } \\
\text { associadas à } \\
\text { intervenção } \\
\text { psicossocial. }\end{array}$ & $\begin{array}{c}\text { A terapia tradicional } \\
\text { chinesa proporcionou um } \\
\text { alívio significativamente } \\
\text { maior no curto prazo }(8 \\
\text { semanas), }(\mathrm{p}=0,02) \mathrm{e} \\
\text { maior redução da } \\
\text { interferência com } \\
\text { atividades sociais } \\
(\mathrm{p}=0,016) .\end{array}$ \\
\hline $\begin{array}{l}\text { Gonzales- } \\
\text { Perez et al. } \\
\text { (2011) }\end{array}$ & $\begin{array}{l}\text { Esse estudo foi realizado } \\
\text { para avaliar a utilidade do } \\
\text { estímulo seco profundo no } \\
\text { tratamento de dor } \\
\text { miofascial. }\end{array}$ & $\begin{array}{l}\text { Agulhamento } \\
\text { à seco }\end{array}$ & $\begin{array}{c}\text { Foi encontrada uma } \\
\text { relação estatisticamente } \\
\text { significativa } 9 \mathrm{p}<0,01) \\
\text { entre a intervenção } \\
\text { terapêutica e a melhora de } \\
\text { dor e movimentos da } \\
\text { mandíbula. }\end{array}$ \\
\hline $\begin{array}{l}\text { Ferreira et } \\
\text { al. (2013) }\end{array}$ & $\begin{array}{l}\text { Este estudo foi realizado } \\
\text { para verificar a eficácia } \\
\text { analgésica do laser diodo } \\
\text { GaAIAs de baixa potência } \\
\text { infravermelho aplicado em } \\
\text { pontos de acupuntura. }\end{array}$ & $\begin{array}{l}\text { Acupuntura } \\
\text { a Laser como } \\
\text { adjunto da } \\
\text { terapia de } \\
\text { tala Oclusal } \\
\text { (Grupo 1) e } \\
\text { Terapia de } \\
\text { tala oclusal } \\
\text { associada a } \\
\text { laser placebo } \\
\text { (Grupo 2). }\end{array}$ & $\begin{array}{l}\text { A redução dos sintomas foi } \\
\text { significativa em ambos os } \\
\text { grupos. As medidas } \\
\text { mostraram valores de } \\
\text { intensidade de dor } \\
\text { significativamente mais } \\
\text { rápidos e mais baixos nos } \\
\text { pacientes que realizaram o } \\
\text { tratamento de laser } \\
\text { acupuntura (p<0,oo2). O } \\
\text { tratamento com laser não } \\
\text { apresentou efeitos } \\
\text { colaterais. }\end{array}$ \\
\hline
\end{tabular}

Fonte: Dados da Pesquisa

DISCUSSÃO

Há muito se sabe que a DTM tem etiologia multifatorial e, em consequência, seu tratamento é complexo e, muitas vezes, demanda da associação de terapias para o controle da dor do paciente. Neste sentido, o tratamento clássico realizada por cirurgiões dentistas envolve o uso de placas oclusais ${ }^{2,4}$ e acompanhamento psicológico ${ }^{9}$ do paciente, porém, há de se destacar o efeito positivo para a melhora da sintomatologia dolorosa quando estes são associados às técnicas da Medicina Tradicional Chinesa. Esta, por sua vez, dispõe de uma série de tratamentos milenares, dentre os quais podem ser citados a acupuntura tradicional $^{2,7,9,10-17}$, acupuntura a laser ${ }^{4,6,8}$, uso de ervas (fitoterapia) ${ }^{9}$ e agulhamento a seco ${ }^{5}$.

A técnica da acupuntura tradicional consiste na inserção de agulhas em pontos específicos de acupuntura, que são mapeados ao longo dos meridianos do corpo humano que almeja conservar a saúde, evitar doenças, curar as enfermidades já instaladas, e corrigir as falhas da perfeita fluência da energia obtendo o equilíbrio, sendo empregada principalmente para o tratamento da dor ${ }^{1,4,11}$. Rosted ${ }^{10}$, Rosted et al. ${ }^{11}$ e Rosted e Jorgensen ${ }^{12}$ avaliaram a efetividade da acupuntura tradicional no tratamento da DTM e de Síndromes Dolorosas. Seus resultados demonstraram que a acupuntura tradicional foi eficaz para essas duas comorbidades. Para Rosted ${ }^{10}$, o uso dessa terapia pode se apresentar como um bom complemento terapêutico para o controle da dor, e para o tratamento da DTM. Enquanto que, para Rosted e Jorgensen ${ }^{12}$ a acupuntura tradicional deve ser indicada em estágios iniciais de Síndromes Dolorosas, uma vez que, a utilização desta técnica apresentou resultados positivos na devolução dos movimentos da mandíbula, que se apresentavam restritos. De acordo com Rosted et al. ${ }^{11}$, a acupuntura se mostrou um método simples, relativamente seguro e potencialmente eficaz e útil na gestão das síndromes dolorosas.

Porém, outros estudos, como o de Shen e Goddard $^{14}$, Smith et al. ${ }^{17}$ e Hunter ${ }^{7}$, que também analisaram o efeito da acupuntura tradicional sobre a sintomatologia da DTM, concluíram que é um tratamento efetivo, mesmo que usado de maneira individual e promoveu a redução da dor decorrente da DTM. Segundo Shen e Goddard ${ }^{14}$, o próprio ato de realizar a acupuntura exerce um efeito placebo que promove melhora do quadro do paciente. Smith et al. ${ }^{17}$ evidencia que a utilização da acupuntura tradicional não se resume a efeitos placebos, mas possui efeitos de analgesia e efeitos fisiológicos. Já Hunter ${ }^{7}$ utilizou como tratamento a combinação de exercício terapia e acupuntura obtendo eficácia no tratamento da DTM, porém, concluiu imprudência se atribuísse a um único fator esse resultado. Dessa forma, definiu a combinação dessas abordagens como eficaz no controle da dor.

O agulhamento a seco é uma técnica de acupuntura que consiste em desativar os pontos de gatilhos com a finalidade de promover o 
relaxamento muscular e consequentemente 0 alívio da dor e desconforto. De acordo com o estudo realizado por González-Perez et al. ${ }^{5}$, a aplicação desta técnica promove melhora na sintomatologia dolorosa e recuperação dos movimentos mandibulares comprometidos em função da dor miofascial. A terapia especifica aparenta ocorrer devido a um rompimento mecânico que acontece durante a inserção das agulhas, todavia, o real mecanismo que resulta na inativação dos pontos de gatilhos dolorosos é desconhecido.

Uma variação da acupuntura tradicional é a técnica da acupuntura a laser, que consiste na estimulação de pontos específicos do corpo com laser de baixa intensidade a fim de atenuar pontos de bloqueio causados pela dor e tensão. Tal modalidade é indolor e atraumática, pois dispensa o uso de agulhas, o que promove uma maior aceitação por parte dos pacientes além de oferecer um menor tempo de tratamento ${ }^{6,8}$. Katsoullis et al. ${ }^{8}$ avaliaram 0 efeito da acupuntura a laser em indivíduos portadores de tendomiopatia dos músculos mastigatórios com intensidade máxima da dor na escala visual analógica (EVA). Nesse estudo, os autores concluíram que este se trata de um tratamento complementar e não invasivo. Seus resultados vão de encontro aos obtidos por Hotta et al. ${ }^{6}$, que avaliaram o efeito do laser de baixa frequência sobre a sintomatologia das DTMs. Os autores observaram melhora tanto da sintomatologia dolorosa quanto na eletromiografia da atividade muscular, contudo não houve melhora estatisticamente significativa dos movimentos mandibulares.

Schimid-Schwap et al. ${ }^{13}$ e Simma et al. ${ }^{16}$ realizaram estudos com o intuito de comparar as técnicas de acupuntura tradicional e a de acupuntura a laser simulada em pacientes com DTM. Em ambos os estudos, os indivíduos de um grupo foram submetidos à terapia com acupuntura tradicional e os do outro grupo, que era placebo, à aplicação da acupuntura a laser simulada, sendo a sintomatologia mensurada através da palpação muscular e escala visual analógica (EVA). Os resultados de SchimidSchwap et al. ${ }^{13}$ demonstraram que apesar de não haver diferenças estatísticas significativas, comparado ao grupo placebo que recebia a laserterapia simulada, o grupo de indivíduos que recebia a acupuntura tradicional apresentou uma redução significativamente maior da dor e melhora na sensibilidade à palpação de todos os músculos. Os autores concluíram que a acupuntura pode ser indicada como tratamento de dor aguda, embora haja a necessidade de estudos com maior número de pacientes para análises a longo prazo. Para Simma et al. ${ }^{16}$, a redução da dor e sensibilidade à palpação também foi superior no grupo que recebeu acupuntura tradicional, inclusive apresentando diferenças estatísticas significativas. Os autores notaram que com apenas uma única sessão pode-se obter alívio das tensões musculares, melhoras na mobilidades da ATM e da dor e concluíram que assim como para SchimidSchwap et al. ${ }^{13}$, a acupuntura tradicional se mostrou efetiva para o tratamento da dor aguda relacionada a DTM.

Um estudo feito por Shin et al. ${ }^{15}$ avaliaram o efeito combinado da acupuntura tradicional e terapia manual. Este último procedimento envolve técnicas de liberação e manipulação muscular que tem como objetivo o relaxamento da musculatura envolvida. Os autores concluíram que tal associação é positiva. Isso foi atribuído ao fato de que a acupuntura resulta em efeitos analgésicos devido ao alívio da dor miofascial e do envolvimento de mecanismos neuro-hormonais. Já a terapia manual, atua relaxando a musculatura mastigatória, resultando em melhora da dor e limitação da abertura de boca.

As placas oclusais são dispositivos removíveis que fornecem um contato interoclusal preciso entre os dentes. Constituem uma terapia conservadora pois são reversíveis e não-invasivas, atuando tanto no fator causal das desordens temporomandibulares (DTMs) quanto na redução dos sintomas. Tais características as permitem fazer com que a articulação temporomandibular adote uma posição articular mais estável, reorganizam a atividade neuromuscular reflexa e protegem dentes e estruturas de suporte de forças anormais que possam criar colapso e/ou desgaste dentário ${ }^{18}$. Bergstrom et al. ${ }^{2}$ e Ferreira et al. ${ }^{4}$ avaliaram a associação de terapia oclusal (placa miorrelaxante) e acupuntura para o tratamento da DTM e ambos obtiveram melhora da sintomatologia dolorosa com a associação dessas técnicas. Ferreira et al. $^{4}$ acrescentam que quando se utiliza a acupuntura a laser os efeitos são ainda melhores. Os autores atribuem esta melhora ao fato de que ao estimular os pontos de gatilho de dor orofacial ST6, SL19, GB20, LI4, LR3, TE3, GB34 e EX-HN3, diminuise a hiperatividade muscular, o que leva a devolução do fluxo sanguíneo normal na musculatura envolvida, desencadeando uma ação analgésica no alivio das dores da ATM, que é potencializada pela ação analgésica puntiforme do laser terapêutico.

A medicina tradicional chinesa se vale de várias formas de terapias, dentre as quais pode- 
se citar o uso de ervas (Fitoterapia), que lança mão do uso de variadas ervas com finalidade terapêutica. Ritenbaugh et al. $^{9}$ avaliaram 0 efeito da acupuntura tradicional associada a ervas e em conjunto com a intervenção psicossocial para o tratamento de DTM crônica. A intervenção psicossocial foi feita través de um protocolo desenvolvido que incluía a educação e percepção sobre a doença, assim como um automonitoramento e o desenvolvimento de um plano pessoal de autocuidado da DTM que contribui para a correção ou melhora de aspectos físicos e psicológicos que possam exacerbar ou manter os sintomas de DTM. Os autores constataram que a terapia tradicional chinesa proporcionou um alívio significativamente maior no prazo de 8 semanas e maior redução da interferência com atividades sociais. Tal fato é atribuído à diminuição do estresse social, que leva a redução da tensão muscular, além também de provocar o alívio da dor local, como efeito do relaxamento muscular, ocasionado pelo estimulo das agulhas inseridas nos pontos de gatilho de dor.

CONCLUSÃO

A partir da literatura pesquisada, conclui se que a acupuntura é uma terapia de suporte complementar no tratamento das disfunções temporomandibulares. Tal modalidade tem se mostrado tão eficiente no controle de dores faciais quanto as terapias convencionais. Assim, a acupuntura e as variações a ela associadas são recomendadas por serem métodos eficazes, não-invasivos e de baixo custo. Contudo, dado o baixo número de pesquisas a longo prazo sobre o tema, sugere-se a realização de mais estudos que possam complementar e confirmar o conhecimento alcançado a fim de comprovar cientificamente a eficácia da acupuntura.

AGRADECIMENTOS

Este artigo recebeu suporte financeiro do Ministério de Educação e Cultura (MEC), através da concessão de bolsas do Programa de Educação Tutorial (PET) aos seus autores e está relacionado às atividades desenvolvidas no projeto de extensão "Oclusão e Desordens Temporomandibulares", vinculado ao grupo PET-Odontologia da UNIFAL-MG.

REFERÊNCIAS

1. Barnes JF. Electronic acupuncture and cold laser therapy as adjuncts to pain treatment. $\mathrm{J}$ Craniomandibular Pract. 1984;2(2):148-52.

2. Bergström I, List T, Magnusson T. A follow-up study of subjective symptoms of temporomandibular disorders in patients who received acupuncture and/or interocclusal appliance therapy 18-20 years earlier. Acta Odontol Scand. 2008;66(2):88-92.

3. Buescher JJ. Temporomandibular joint disorders. Am Fam Physician. 2007;76(10): 1477-82.

4. Ferreira LA, de Oliveira RG, Guimarães JP, Carvalho AC, De Paula MV. Laser acupuncture in patients with temporomandibular dysfunction: a randomized controlled trial. Lasers Med Sci. 2013;28(6):1549-58.

5. Gonzalez-Perez LM, Infante-Cossio $P$, Granados-Nuñez M, Urresti-Lopez FJ. Treatment of temporomandibular myofascial pain with deep dry needling. Med Oral Patol Oral Cir Bucal. 2012;17(5):e781-85.

6. Hotta PT, Hotta TH, Bataglion C, Bataglion SA, de Souza Coronatto EA, Siéssere $S$, et al. Emg analysis after laser acupuncture in patients with temporomandibular dysfunction (TMD). Implications for practice. Complement Ther Clin Pract. 2010;16(3):158-60.

7. Hunter EK. Integration of rehabilitation and acupuncture in the treatment of a professional musician with temporomandibular joint dysfunction. Acupunct Med. 2011;29(4): 298-301.

8. Katsoulis J, Ausfeld-Hafter B, Windecker-Gétaz I, Katsoulis K, Blagojevic N, Mericske-Stern R. Laser acupuncture for myofascial pain of the masticatory muscles. A controlled pilot study. Schweiz Monatsschr Zahnmed. 2010;120(3): 213-25.

9. Ritenbaugh C, Hammerschlag R, Dworkin SF, Aickin MG, Mist SD, Elder CR, et al. Comparative effectiveness of traditional Chinese medicine and psychosocial care in the treatment of temporomandibular disordersassociated chronic facial pain. J Pain. 2012; 13(11):1075-89.

10. Rosted P. Introduction to acupuncture in dentistry. Br Dent J. 2000;189(3):136-40.

11. Rosted P, Bundgaard M, Pedersen AM. The use of acupuncture in the treatment of temporomandibular dysfunction--an audit. Acupunct Med. 2006;24(1):16-22.

12. Rosted $P$, Jørgensen V. Acupuncture treatment of pain dysfunction syndrome after dental extraction. Acupunct Med. 2002;20(4):191-92.

13. Schmid-Schwap M, Simma-Kletschka I, Stockner A, Sengstbratl M, Gleditsch J, Kundi $M$, et al. Oral acupuncture in the therapy of craniomandibular dysfunction syndrome -- a randomized controlled trial. Wien Klin Wochenschr. 2006;118(1-2):36-42.

14. Shen YF, Goddard G. The short-term effects of acupuncture on myofascial pain patients after clenching. Pain Pract. 2007;7(3):256-64.

15. Shin BC, $\mathrm{Ha} \mathrm{CH}$, Song YS, Lee MS. Effectiveness of combining manual therapy and acupuncture on temporomandibular joint 
dysfunction: a retrospective study. Am J Chin Med. 2007;35(2):203-8.

16. Simma I, Gleditsch JM, Simma L, Piehslinger E. Immediate effects of microsystem acupuncture in patients with oromyofacial pain and craniomandibular disorders (CMD): a doubleblind, placebo-controlled trial. Br Dent J. 2009; 207(12):E26.

17. Smith P, Mosscrop D, Davies S, Sloan P, Al-Ani $Z$. The efficacy of acupuncture in the treatment of temporomandibular joint myofascial pain: a randomised controlled trial. J Dent. 2007; 35(3):259-67.

18. Okeson JP. Tratamento das desordens temporomandibulares e oclusão. 6. ed. Rio de Janeiro: Elsevier; 2008.

\section{CONFLITO DE INTERESSES}

Os autores declaram não haver conflitos de interesse

AUTOR PARA CORRESPONDÊNCIA

Mateus Silva Beker dos Reis

Avenida Afonso Pena 1613a 37135-106 Alfenas - MG, Brasil

E-mail: mateus.beker@sou.unifal-mg.edu.br

Submetido em $27 / 11 / 2020$

Aceito em 24/09/2021 